\title{
Duty Rostering for Physicians at a Department of Orthopedics and Trauma Surgery
}

\author{
Clemens Thielen ${ }^{\mathrm{a}, *}$ \\ ${ }^{a}$ Department of Mathematics, University of Kaiserslautern, Paul-Ehrlich-Str. 14, \\ D-67663 Kaiserslautern, Germany
}

\begin{abstract}
This paper presents a case study of duty rostering for physicians at a department of orthopedics and trauma surgery. We provide a detailed description of the rostering problem faced and present an integer programming model that has been used in practice for creating duty rosters at the department for more than a year. Using real world data, we compare the model output to a manually generated roster as used previously by the department and analyze the quality of the rosters generated by the model over a longer time span. Moreover, we demonstrate how unforeseen events such as absences of scheduled physicians are handled.
\end{abstract}

Keywords: personnel scheduling, rostering, physicians, integer programming

\section{Introduction}

Scheduling nurses and physicians is a central task within personnel planning in hospitals and has a large impact both on the efficient operation of a hospital as well as on employee satisfaction. The creation of duty rosters for physicians is particularly important since physicians are often a scarce resource for hospitals [1] and usually very difficult to replace [2]. This has important implications for the scheduling process since it leads to a partial transfer of power from management to physicians and increases the importance of respecting the physicians' preferences [3]. Hence, achieving a fair

\footnotetext{
${ }^{*}$ Corresponding author. Fax: +49 (631) 205-4737. Phone: +49 (631) 205-4590

Email address: thielen@mathematik.uni-kl.de (Clemens Thielen)
} 
distribution of duties and respecting the physicians' individual preferences are crucial aspects in designing duty rosters for physicians. In addition, numerous requirements on physician rosters resulting, e.g., from mandatory rest periods, demand fluctuation, and a high relevance of different experience levels due to continued medical education make duty rostering for physicians particularly challenging and substantially different from many related personnel scheduling problems such as nurse rostering.

Even though physician scheduling has received growing attention in the scheduling and operations research literature within the last years [3], the schedules and duty rosters used in practice are often still created manually by an experienced physician [4], which not only leads to unfairness and violations of labor regulations, but also requires a large amount of a highly trained physician's valuable time. Thus, there is still a lot of potential for practical applications of operations research methods in physician scheduling.

This paper presents a case study of duty rostering for physicians at a large department of orthopedics and trauma surgery. We provide a detailed description of the rostering problem faced as well as the resulting integer programming (IP) model, which has been used in practice for more than a year. Using real world data from the practice partner, we show that the model generates high-quality rosters and improves greatly upon the previously used manual rostering approach. Moreover, we demonstrate how unforeseen events such as absences of scheduled physicians due to illness are handled.

\subsection{Related Work}

Workforce or personnel scheduling is a classical field within the scheduling and operations research literature. For a recent survey on this general topic, we refer to Van den Bergh et al. [5].

Many papers focusing on personnel scheduling in the health care sector consider nurse scheduling, which has been reviewed, e.g., in $[6,7,8]$. Nurse scheduling models, however, can usually not handle the more complex constraints arising in most practical applications of physician scheduling.

An extensive survey on physician scheduling has recently been conducted by Erhard et al. [3], who list 68 relevant publications dealing with various aspects of the problem. However, only few of the advanced mathematical models developed in these publications are successfully used in practice. Duty rostering models that are used in every-day practice include the models by

Carter and Lapierre [9] and Fügener et al. [10]. Carter and Lapierre [9] 
analyze the schedules for emergency room physicians used in six hospitals and develop tabu search heuristics for computing improved schedules, which have been adopted for practical use by two of the hospitals. Fügener et al. [10] present two mixed integer programming (MIP) models for assigning physicians to duties and subsequently to workstations at a department of anesthesiology. Their models are used in practice by a large teaching hospital in Germany.

Respecting the physicians' individual preferences and achieving a fair distribution of the workload are important aspects considered in the physician scheduling literature. As Erhard et al. [3] note, these aspects influencing the employees' satisfaction with the generated duty rosters are particularly important in physician scheduling since physicians are highly qualified personnel and usually very difficult to replace. Respecting the physicians' individual preferences usually means assigning as many physicians as possible to requested duties while avoiding the assignment of physicians to duties declared as undesired. Workload fairness is often considered by trying to achieve an equal distribution both of the overall workload (e.g., the total number of duties or working hours) and of unpopular duties such as duties on weekends or public holidays [11].

While re-planning in case of unforeseen events (such as absence of physicians due to illness) is also an important factor in practice, re-planning aspects have been largely neglected in the physician scheduling literature so far. To the best of our knowledge, the only model that considers re-planning explicitly is due to Gross et al. [12]. Building on the model of Fügener et al. [10], they present a MIP model for creating updated duty and workstation rosters in the case of absences of scheduled personnel.

The model presented in this paper is based on a preliminary duty rostering model developed with students in a bachelor's thesis [13] and a student project [14].

\subsection{Our Contribution}

We present a duty rostering model for physicians that has been used in practice at a large department of orthopedics and trauma surgery for more than a year.

Using real world data, we demonstrate that the generated duty rosters clearly outperform the rosters generated by the previous manual rostering approach in all important metrics. Moreover, we analyze the quality of the 
duty rosters computed by our model from a practical point of view over a longer time span.

Our approach incorporates re-planning in case of unforeseen absences of scheduled physicians by generating a list of possible substitutes for each duty on each day. This represents a new approach for dealing with this important aspect of physician scheduling and has turned out to be very useful in practice.

In addition to providing a detailed, practical case study of physician scheduling that demonstrates the effectiveness of using operations research methods in this field, we believe that many parts of our work will also be useful in advancing the general state of the art in physician scheduling models for departments in the area of orthopedics and trauma surgery. Even though our model is designed specifically for the rostering problem faced at the partner department, many parts of the model should be easily transferable to similar settings in other hospitals' orthopedics and trauma surgery departments - a type of department whose duty rostering problems have received only very limited attention in the physician scheduling literature so far.

\section{Problem Description}

In this section, we describe the rostering problem presented to us by the department. Moreover, we shortly describe the previous (manual) rostering approach used as well as its shortcomings.

We are given a set of about 50 physicians, who are partitioned into residents (who are still in their six years of practical training) and fellows (who have already completed their practical training). There are 12 different teams (e.g., trauma, spine, shoulder, or hand surgery) each consisting of 2-9 physicians. Each fellow is permanently assigned to exactly one these teams. Each resident, on the other hand, is either permanently assigned to one team or serves as a float physician that can be flexibly assigned to a different team from a given subset of the teams on each work day (where the term "work day" refers to the days from Monday to Friday that are not public holidays). While the permanent assignments of physicians to teams are given as input, the flexible assignment of the float physicians to teams on each work day is to be determined during the duty rostering process in order to compensate violated team staffing requirements (as will be detailed below). The normal working hours of the physicians in the teams are from 7:15 am to 4:00 pm 
on each work day, while no work is performed in the teams on weekends or public holidays.

The main part of the duty rostering problem (besides the flexible assignment of the float physicians to teams) consists of assigning physicians to five different night duties D1, ., D5 and one late duty. ${ }^{1}$ On each day of the planning period (which is usually two months, but sometimes slightly shorter or longer due to holiday periods), exactly one physician has to be assigned to each of these duties. The night duties start directly after the normal working hours (i.e., at 4:00 pm) on work days or between 8:30 am and 9:00 am on weekends and public holidays and end the following morning. The late duty lasts from 3:00 pm to 11:30 pm on work days and from 1:00 pm to 11:00 pm on weekends and public holidays.

The duties require different experience levels, so that each duty should only be assigned to physicians with a matching level of experience (e.g., two of the night duties should only be assigned to fellows and two other night duties should be assigned only to residents in their first or second year). The set of physicians eligible for a certain duty will be referred to as the duty group of this duty. Most physicians are part of two different duty groups (i.e., eligible for two different duties), but some only belong to a single duty group.

The assignment of the six duties to physicians interacts with the work performed in the teams since assigning a duty to a physician can result in the physician being absent in her ${ }^{2}$ team either on the day of the duty or on the following day. This has to be taken into account when assigning the duties because of so-called team staffing requirements. These team staffing requirements are given by a team dependent maximum total number of physicians (residents and fellows) that is allowed to be absent in each team on each work day. Additionally, there is a team dependent maximum number of fellows that can be absent in each team on each work day.

Since the surgical schedule of each team is usually full on almost all work

\footnotetext{
${ }^{1}$ Throughout the paper, we will use the term "duty" only when referring to the five night duties or the late duty. In particular, the work performed by the physicians in the teams on work days will not be referred to as "duty".

${ }^{2}$ Even though there are male as well as female physicians within the department, we will always refer to a single physician as female throughout the paper for the sake of comprehensiveness.
} 
days and slight demand fluctuations are not yet know when the duty roster for the two-month planning period is created, these numbers are the same for each day of the planning period and are chosen such that the full surgical program can still be handled with the remaining number of physicians / fellows in each team. While violated team staffing requirements can be compensated by assigning float physicians to the corresponding teams when creating the duty roster, this is not possible for violated fellow staffing requirements since only residents can be designated as float physicians. Moreover, since no work is performed in the teams on weekends and public holidays and only the physicians on duty are present, there are no staffing requirements for teams on these days. Furthermore, during certain leave periods (around Easter and Christmas), half of the physicians are on leave and the surgical schedule is reduced. Hence, the maximum total numbers of absent physicians in all teams are higher on all work days of these leave periods and no maximum numbers of absent fellows are required.

Absences of physicians in the teams on work days, which are relevant for the team staffing requirements, occur either as planned absences (vacations, conference visits, etc.) or because one of the duties is assigned to a physician. The absences of a physician (on the day of the duty or the following day) caused when the physician is assigned a duty are provided in Table 1. As can also be seen in Table 1, some duties cause the assigned physician to start work in her team later (10:45 am instead of 7:15 am) on either this day or the following day (if the corresponding day is a work day), e.g., in order to guarantee a sufficient rest period after a late duty. Physicians starting late are not considered as absent in their team on the corresponding day, but late starts are undesired in case that the staffing of the team is already close to its minimum.

In addition to absences and late starts in her team on work days, Table 1 also provides an overview of the implications that the assignment of a duty to a physician and the resulting rest periods have on the duties this physician can be assigned on the following day. For example, no duty (late or night) can be assigned to a physician on the day after a night duty D1, D2, D3, or D5 since this day is required as a rest period. In contrast, duty D4 is a stand-by duty. This means that, while it is undesirable to assign a duty to a physician on the day after a night duty D4, any duty is possible on such a day. On the day after a late duty, any duty is possible as well. Moreover, it is desirable to assign the late duty to the same physician on several consecutive 


\begin{tabular}{|c|c|c|c|c|c|}
\hline \multirow{2}{*}{$\begin{array}{c}\text { Duty on } \\
\text { day } t\end{array}$} & \multicolumn{2}{|c|}{ Presence in team } & \multicolumn{2}{|c|}{ Start of work in team } & \multirow{2}{*}{$\begin{array}{c}\text { Duty on } \\
\text { day } t+1 \text { possible? }\end{array}$} \\
\hline & Day $t$ & Day $t+1$ & Day $t$ & Day $t+1$ & \\
\hline D1 & present & absent & 10:45 am & - & no \\
\hline D2 & present & absent & $7: 15 \mathrm{am}$ & - & no \\
\hline D3 & present & absent & 10:45 am & - & no \\
\hline $\mathrm{D} 4$ & present & present & $7: 15$ am & $7: 15 \mathrm{am}$ & yes (but undesired) \\
\hline D5 & present & absent & 7:15 am & - & no \\
\hline Late & absent & present & - & 10:45 am & yes (Late desired) \\
\hline
\end{tabular}

Table 1: Implications of assigning a duty to a physician on the presence in her team on work days and on possible duties on the following day.

days in order to avoid a constant change between working in the team during the day and working the late duty.

In order to specify their planned absences and preferences for each day, each physician specifies a number in $\{1,2,3,4\}$ for each day of the planning period (and the first day of the following planning period) with the following meaning:

- 1 - present and duty request on this day

- 2 - present, but no specific preference for this day

- 3 - present, but requesting not to be assigned any duty on this day

- 4 - planned absence on this day

Alternatively, a physician can request a specific duty she is eligible for on each day by specifying the name of this duty instead of one of the numbers. If a physician specifies a planned absence (entry 4) for a day, she cannot be assigned to any duty (including the late duty) on this day or the day before. The data about the preferences / absences is submitted as an Excel spreadsheet column by each physician about two weeks before the start of the planning period. The rules imposed on the preferences for each physician state that only work days previously granted as planned absence can be marked with a 4 and at most half of the remaining work days can be marked with a 3. Public holidays or days on weekends can be marked with a 4 only if a planned absence has previously been granted on at least one of the adjacent work days, and again only half of the remaining days can be marked with a 3 . 
The goal of the rostering process is to generate a feasible duty roster respecting all the above conditions while at the same time respecting the physicians' individual preferences, achieving a fair assignment of duties (both within each duty group and overall), and satisfying certain additional desirable properties. Moreover, if possible, a float physician should be assigned to a team on each work day where this is necessary in order to avoid or reduce the violation of a team staffing requirement. If some float physicians are not needed in any team on some days, no assignment of these float physicians to teams needs to be specified for these days.

Respecting the physicians' individual preferences means to fulfill as many duty requests as possible while minimizing the number of duties assigned to physicians that requested not to be assigned to any duty on the corresponding day.

A fair assignment of duties within each duty group means that each physician should be assigned a fair number of duties of the corresponding type. Since duties on weekends and public holidays (including night duties from Friday to Saturday morning or from the day before a public holiday to the morning of the holiday) are unpopular among most physicians, a fair assignment should be achieved separately for the duties during the week and for the ones on weekends / public holidays. Furthermore, the number of days present (not on planned absence) of a physician and the total number of duty groups she is part of should be taken into account when determining the "fair" number of duties for each physician within each duty group. For example, this ensures that a physician with a longer vacation in the planning period receives less duties than a physician of the same duty group who is present for the whole period. Moreover, a physician that is part of only one duty group receives more duties within this duty group than a colleague who additionally belongs to another duty group.

In order to also achieve a fair allocation of duties overall, the numbers of duties assigned to a physician in her different duty groups should be linked. This means that a physician receiving a little more duties than desired in one duty group (which must be allowed since it is general not possible to assign exactly the same number of duties per day not absent to each physician in a duty group) should receive a little less duties than desired in her other duty group. Additionally, no physician should be assigned too many duties on Saturdays or during leave periods in total since these duties are particularly unpopular. 
Some physicians, however, are excluded from the fair allocation rules (either during the week, for weekends, or for both parts of the fair allocation) and should receive a fixed number of duties of a given type per month. For instance, single parents are usually excluded from the fair allocation (at least until their children reach a certain age) and are only given a small, fixed number of duties.

Other desirable properties of a good duty roster include that the night duties of each physician should not be too close together, so the physician has at least two (and preferably even three) days without night duties in between any two night duties whenever possible.

\subsection{Previous Rostering Procedure}

Before switching to the rostering method presented in this paper, the duty rosters for the department had been generated manually by an experienced physician (called the scheduler in the following). This process took the scheduler two to three full work days of time. The general approach of the scheduler can be described as follows: In a first step, as many physicians as possible were assigned requested duties. In the second step, the remaining duties were assigned in order to achieve a fair distribution of duties within each duty group while trying to keep the team staffing requirements in mind and avoiding undesired duties (i.e., duties on days marked with a 3 by the corresponding physician). The assignment of float physicians to teams on work days was determined spontaneously in the morning without prior planning by the scheduler.

While this approach led to a good number of duty requests being fulfilled, the resulting rosters often violated the team staffing requirements and did not achieve a fair distribution of the duties in the different duty groups. Moreover, it often happened that physicians were assigned to duties outside their duty groups - most likely in order to assign duties on days where only few physicians were available. While this had no legal implications (since the experience levels required for the different duties are not statutory but part of the department's policy), it still resulted in dissatisfaction of the physicians with the generated rosters and in reduced efficiency of the physicians on duty.

The amount of time required for generating the rosters and the shortcomings of the manually generated rosters mentioned above were the department's main reasons for switching to an automated scheduling approach via an IP model. Reducing the amount of violated team staffing requirements 
was particularly important for the department since these violations often led to surgeries having to be postponed to the next day or physicians having to violate the mandatory rest periods. Moreover, a fairer assignment of the duties in the different duty groups was desired in order to improve the physicians' satisfaction with the generated rosters.

\section{Model}

We now present the model used to assign the six different duties to the physicians and the float physicians to the teams. In this model, the conditions and objectives presented in the previous section are modeled in great detail, which has been achieved through close collaboration with the department and the scheduler in a constant feedback and adaption process. In particular, the team staffing requirements and the conditions on the fair allocation of duties (both within each duty group and overall) are modeled in great detail in order to ensure the generation of rosters that result in good working conditions in the teams and physicians being satisfied with the generated rosters.

We use the following notation:

\section{Sets:}

$\begin{array}{ll}P & \text { set of physicians (index } p \text { ) } \\ D & \text { set of duties D1, D2, D3, D4, D5, Late (index } d \text { ) } \\ P_{d} & \text { subset of physicians belonging to the duty group of duty } d \in D \\ D_{p} & \text { subset of duties to whose duty group physician } p \in P \text { belongs } \\ P^{\text {fellow }} & \text { subset of fellows } \\ P^{\text {float }} & \text { subset of float physicians } \\ P^{\text {fix }} & \text { subset of physicians with a fixed number of duties on work } \\ P^{\text {fix, we }} & \text { days } \\ G & \text { subset of physicians with a fixed number of duties on weekends } \\ P_{g} & \text { subset of physicians belonging to team } g \in G\end{array}$


$G_{p} \quad$ subset of teams to which float physician $p \in P^{\text {float }}$ can be assigned.

$P_{g}^{\text {float }}$

T

$T^{\mathrm{WE}}$

$T^{\mathrm{WE}+}$

$T^{\text {Sat }}$

$T^{\text {leave }}$

$T^{\text {leave,work }}$

$T_{1}$

$T_{2}$

\section{Parameters:}

$a(p, t)$

$\operatorname{size}(g)$

$\operatorname{abs}(g, t)$

$\operatorname{abs}^{f}(g, t)$

$\operatorname{abs}^{l}(g, t)$

$\operatorname{nd}(p, d)$

$\operatorname{nd}^{\mathrm{WE}}(p, d)$ subset of float physicians that can be assigned to team $g \in G$

set of days $1, \ldots, n$ of the planning period (index $t$ )

subset of days on weekends or public holidays

subset of days on weekends or public holidays extended by the days before

subset of all Saturdays

subset of all days during leave periods

subset of all work days during leave periods

subset of all days of month 1

subset of all days of month 2
1 if physician $p \in P$ is absent on day $t \in T \cup\{n+1\}$, 0 otherwise

number of physicians in team $g \in G$

maximum total number of absent physicians in team $g \in G$ on work day $t \in T \backslash\left(T^{\mathrm{WE}} \cup T^{\text {leave,work }}\right)$

maximum number of absent fellows in team $g \in G$ on work day $t \in T \backslash\left(T^{\mathrm{WE}} \cup T^{\text {leave,work }}\right)$

maximum total number of absent physicians in team $g \in G$ on work day $t \in T^{\text {leave,work }}$ during a leave period

desired number of duties of type $d \in D$ of physician $p \in$ $P_{d} \backslash P^{\mathrm{fix}}$ on work days

desired number of duties of type $d \in D$ of physician $p \in$ $P_{d} \backslash P^{\mathrm{fix}, \mathrm{WE}}$ on weekends and public holidays 
$\operatorname{duty}^{\text {fix }}(p) \quad$ specific duty to be assigned to physician $p \in P^{\text {fix }}$ on work days

$\operatorname{duty}^{\text {fix }, \mathrm{WE}}(p) \quad$ specific duty to be assigned to physician $p \in P^{\mathrm{fix}, \mathrm{WE}}$ on weekends and public holidays (including the days before in case this is a night duty)

$\operatorname{nd}^{\text {fix }}(p)$

fixed number of duties on work days to be assigned to physician $p \in P^{\text {fix }}$

$\operatorname{nd}^{\mathrm{fix}, \mathrm{WE}}(p)$

fixed number of duties on weekends and public holidays (including the days before if $\operatorname{duty}^{\mathrm{fix}, \mathrm{WE}}(p)$ is a night duty) to be assigned to physician $p \in P^{\text {fix }, \mathrm{WE}}$

$i(p, g, t) \quad 1$ if float physician $p \in P^{\text {float }}$ has been assigned to team $g \in$ $G_{p}$ on work day $t \in T \backslash T^{\mathrm{WE}}$ during the preprocessing phase, 0 otherwise

In order to achieve a fair allocation of duties of each type $d \in D$ (i.e., in each duty group), the desired numbers $\operatorname{nd}(p, d)$ and $\mathrm{nd}^{\mathrm{WE}}(p, d)$ of duties of each physician are calculated proportionally to the quotient of the number of days of the planning period on which the corresponding physician is present (not on planned absence) and the number of duty groups the physician is part of. More precisely, denoting the total number of work days for duty $d \in D$ by $\operatorname{wd}(d)$ (which includes Fridays and days before public holidays only for the late duty), the number of days physician $p \in D_{p}$ is present during the planning period by present $(p)$, and the number of duty groups physician $p$ is part of by $\operatorname{dgs}(p)$, we set

$$
\operatorname{nd}(p, d):=\left(\operatorname{wd}(d)-\sum_{\substack{p^{\prime} \in P^{\text {fix }} \\ \text { duty }^{\text {fix }}\left(p^{\prime}\right)=d}} \operatorname{nd}^{\text {fix }}\left(p^{\prime}\right)\right) \cdot \frac{\operatorname{present}(p) / \operatorname{dgs}(p)}{\sum_{p^{\prime \prime} \in P_{d} \backslash P^{\text {fix }}} \operatorname{present}\left(p^{\prime \prime}\right) / \operatorname{dgs}\left(p^{\prime \prime}\right)} .
$$

Similarly, denoting the total number of days on weekends and public holidays for duty $d \in D$ (including Fridays and the days before public holidays for the night duties) by $\operatorname{wph}(d)$, we set

$$
\operatorname{nd}^{\mathrm{WE}}(p, d):=\left(\operatorname{wph}(d)-\sum_{\substack{p^{\prime} \in P_{\mathrm{fix}, \mathrm{WE}:} \\ \text { duty } \mathrm{fix}_{\mathrm{WE}}\left(p^{\prime}\right)=d}} \operatorname{nd}^{\mathrm{fix}, \mathrm{WE}}\left(p^{\prime}\right)\right) \cdot \frac{\operatorname{present}(p) / \operatorname{dgs}(p)}{\sum_{p^{\prime \prime} \in P_{d} \backslash P^{\mathrm{fix}, \mathrm{WE}}} \operatorname{present}\left(p^{\prime \prime}\right) / \operatorname{dgs}\left(p^{\prime \prime}\right)} .
$$


Note that the numbers $\operatorname{nd}(p, d)$ and $\mathrm{nd}^{\mathrm{WE}}(p, d)$ will, in general, not be integers. Therefore, in the constraints ensuring a fair distribution of duties in the model, these numbers are rounded up or down to the next integer and some slack is introduced to allow necessary (but small) deviations from the desired numbers of duties for each physician. Also note that the above definitions ensure that the sum of the desired numbers of duties of all physicians in a duty group always equals the number of duties to be assigned after the duties to be performed by the physicians with a fixed number of duties have been subtracted (no matter how many duty groups the physicians belong to and how many duties are performed by physicians with a fixed number of duties). Moreover, even though physicians with more vacation days during the planning period receive less duties, this balances out over the year to an equal distribution since every physician has the same number of vacation days per year. Part-time contracts, however, are automatically taken into account by our approach since each part-time physician of the department has a certain number of days of planned absence each week (e.g., she might never work on Thursdays or Fridays), which results in less duties being assigned to her.

While a fair allocation of duties is achieved through the constraints of the model, the physicians' preferences - given by the submitted duty requests (entry 1 or name of specific duty requested) and the days marked as undesired for being on duty (entry 3) - are modeled in the objective function. Each fulfilled duty request yields a reward in the objective function, whereas each undesired duty results in a penalty. The exact values of these rewards / penalties are specified in the input (see the description of the objective function below and the table of decision variables and objective function coefficients provided in the appendix). Here, the reward for each fulfilled duty request and the penalty for each undesired duty are independent of the particular physician. Moreover, the reward for a fulfilled duty request is the same no matter whether the physician requested any duty on the corresponding day (entry 1) or specifically requested the assigned duty.

Before generating the IP, several preprocessing steps are applied to check consistency of the input data. For example, it is checked whether all physicians have respected the rule of marking at most half of the days they are not absent with a 3 (request not to be assigned any duty on this day). If some physicians do not respect this rule, a warning message with the names of the physicians is output and their preferences are ignored (i.e., all entries 1, 3, and requests for specific duties of are replaced by an entry 2 ). 
Moreover, it often happens that the maximum numbers of absent physicians and / or fellows are already violated in some teams on some work days due to planned absences before any duties are assigned. If this is the case and the violations can be avoided / reduced by assigning float physicians to the corresponding teams on the days in question, a fixed assignment of these float physicians to the teams is made. If some team staffing requirements are still violated afterwards because there are not enough float physicians available on some days, the maximum numbers of absent physicians / fellows in the corresponding teams are increased for these days. ${ }^{3}$ Hence, only violations of team staffing requirements resulting from the assignment of duties are relevant in the IP model (but also previously existing violations are reported in the output).

As in many duty rostering models (see, e.g., [3]), the constraints of the IP model are partitioned into hard constraints and soft constraints. Soft constraints can be violated, but each violation is penalized in the objective function (alternatively, for some constraints, a reward is given whenever the constraint is satisfied). The exact value of the penalty / reward for each soft constraint is specified in the input.

Below, we describe the objective function and all constraints of the model verbally using the sets and parameters introduced above. The exact formulation of all constraints of the model in the IP is provided in the appendix. The linear objective function (described in the following paragraph and given by the objective function coefficients of the decision variables provided in Table 4 in the appendix) is not written out explicitly due to its size.

\section{Objective function:}

The linear objective function to be maximized consists of the rewards obtained from fulfilled duty requests and certain soft constraints minus the penalties obtained from undesired duties and the remaining soft constraints. The coefficients of all variables appearing in the objective function are provided in the input of the model and can be easily changed by the practice partner. A table of all decision variables, their coefficients in the objective function, and the corresponding coefficient values that are normally used by the practice partner is provided in Table 4 in the appendix.

\footnotetext{
${ }^{3}$ Thus, the parameters abs $(g, t), \operatorname{abs}^{f}(g, t)$, and $\operatorname{abs}^{l}(g, t)$ used in the model depend on the day $t$, even though the maximum numbers of absent physicians / fellows provided in the input are initially independent of $t$.
} 


\section{Hard constraints:}

Assignment constraints

(1) Each duty $d \in D$ is assigned to exactly one physician $p \in P_{d}$ on each day $t \in T$.

(2) Each physician $p \in P$ is assigned at most one duty $d \in D_{p}$ on each day $t \in T$.

Respecting planned absences

(3) No duty is assigned to a physician $p \in P$ on day $t \in T$ if $p$ is absent on day $t$ (i.e., if $a(p, t)=1$ ).

(4) No duty is assigned to a physician $p \in P$ on day $t \in T$ if $p$ is absent on day $t+1$ (i.e., if $a(p, t+1)=1)$.

No duty on the day after a night duty (except for D4)

(5) If physician $p \in P$ is assigned a night duty D1, D2, D3, or D5 on day $t \in$ $T \backslash\{n\}$, she cannot be assigned any duty on day $t+1$.

Assignment of float physicians to teams on work days

(6) Each float physician $p \in P^{\text {float }}$ is assigned to at most one team $g \in G_{p}$ on each work day $t \in T \backslash T^{\mathrm{WE}}$.

(7) If float physician $p \in P^{\text {float }}$ is absent on work day $t \in T \backslash T^{\mathrm{WE}}$ (i.e., if $a(p, t)=1)$, she cannot be assigned to any team on day $t$.

(8) If float physician $p \in P^{\text {float }}$ is assigned a night duty D1, D2, D3, or D5 on day $t \in T \backslash\{n\}$, she cannot be assigned to any team on day $t+1$.

(9) If float physician $p \in P^{\text {float }}$ has been assigned to team $g \in G_{p}$ on work day $t \in T \backslash T^{\mathrm{WE}}$ during the preprocessing phase (i.e., if $i(p, g, t)=1$ ), this assignment is respected.

Fair allocation of duties within each duty group

(10) For each night duty $d \in D \backslash\{$ Late $\}$, each physician $p \in P_{d} \backslash P^{\text {fix }}$ is assigned at least $\lceil\operatorname{nd}(p, d)\rceil-1$ duties of type $d$ on work days $t \in$ $T \backslash T^{\mathrm{WE}+}$. 
(11) For each night duty $d \in D \backslash\{$ Late $\}$, each physician $p \in P_{d} \backslash P^{\text {fix }}$ is assigned at most $\lfloor n d(p, d)\rfloor+1$ duties of type $d$ on work days $t \in$ $T \backslash T^{\mathrm{WE}+}$.

(12) For each night duty $d \in D \backslash\left\{\right.$ Late , each physician $p \in P_{d} \backslash P^{\mathrm{fix}, \mathrm{WE}}$ is assigned at least $\left\lceil\mathrm{nd}^{\mathrm{WE}}(p, d)\right\rceil-1$ duties of type $d$ on days $t \in T^{\mathrm{WE}+}$ on weekends, public holidays, or the days before.

(13) For each night duty $d \in D \backslash\{$ Late $\}$, each physician $p \in P_{d} \backslash P^{\mathrm{fix}, \mathrm{WE}}$ is assigned at most $\left\lfloor\operatorname{nd}^{\mathrm{WE}}(p, d)\right\rfloor+1$ duties of type $d$ on days $t \in T^{\mathrm{WE}+}$ on weekends, public holidays, or the days before.

(14) Each physician $p \in P_{\text {Late }} \backslash P^{\text {fix }}$ in the duty group of the late duty is assigned at least $\lfloor$ nd $(p$, Late $)\rfloor-1$ late duties on work days $t \in T \backslash T^{\mathrm{WE}}$.

(15) Each physician $p \in P_{\text {Late }} \backslash P^{\text {fix }}$ in the duty group of the late duty is assigned at most $\lfloor\operatorname{nd}(p$, Late $)\rfloor+2$ late duties on work days $t \in T \backslash T^{\mathrm{WE}}$.

(16) Each physician $p \in P_{\text {Late }} \backslash P^{\text {fix, } \mathrm{WE}}$ in the duty group of the late duty is assigned at least $\left\lfloor\mathrm{nd}^{\mathrm{WE}}(p\right.$, Late $\left.)\right\rfloor-1$ late duties on days $t \in T^{\mathrm{WE}}$ on weekends and public holidays.

(17) Each physician $p \in P_{\text {Late }} \backslash P^{\text {fix, } \mathrm{WE}}$ in the duty group of the late duty is assigned at most $\left\lfloor n d^{\mathrm{WE}}(p\right.$, Late $\left.)\right\rfloor+2$ late duties on days $t \in T^{\mathrm{WE}}$ on weekends and public holidays.

Fair allocation of duties overall

(18) Each physician $p \in P \backslash P^{\text {fix }}$ is assigned at least $\left\lceil\sum_{d \in D_{p}} \operatorname{nd}(p, d)\right\rceil-2$ duties in total on work days of the corresponding duties $\left(T \backslash T^{\mathrm{WE}}+\right.$ for night duties and $T \backslash T^{\mathrm{WE}}$ for the late duty).

(19) Each physician $p \in P \backslash P^{\mathrm{fix}}$ is assigned at most $\left\lfloor\sum_{d \in D_{p}} \operatorname{nd}(p, d)\right\rfloor+2$ duties in total on work days of the corresponding duties $\left(T \backslash T^{\mathrm{WE}}+\right.$ for night duties and $T \backslash T^{\mathrm{WE}}$ for the late duty).

(20) Each physician $p \in P \backslash P^{\mathrm{fix}, \mathrm{WE}}$ is assigned at least $\left\lceil\sum_{d \in D_{p}} \mathrm{nd}^{\mathrm{WE}}(p, d)\right\rceil-$ 1 duties in total on weekends and public holidays (including the days before for the night duties). 
(21) Each physician $p \in P \backslash P^{\mathrm{fix}, \mathrm{WE}}$ is assigned at most $\left\lfloor\sum_{d \in D_{p}} \mathrm{nd}(p, d)^{\mathrm{WE}}\right\rfloor+$ 1 duties in total on weekends and public holidays (including the days before for the night duties).

At most two duties on Saturdays for each physician

(22) Each physician $p \in P \backslash P^{\text {fix,WE}}$ should be assigned at most two duties on Saturdays in total.

Numbers of duties of physicians with fixed numbers of duties

(23) Each physician $p \in P^{\text {fix }}$ with a fixed number $n d^{\text {fix }}(p)$ of duties on work days is assigned exactly this number of duties of type duty ${ }^{\mathrm{fix}}(p)$ (and no other duties) on work days each month.

(24) Each physician $p \in P^{\text {fix,WE}}$ with a fixed number $\mathrm{nd}^{\mathrm{fix}, \mathrm{WE}}(p)$ of $\mathrm{du}-$ ties on weekends and public holidays (including the days before if duty $^{\mathrm{fix}, \mathrm{WE}}(p)$ is a night duty) is assigned exactly this number of duties of type $\operatorname{duty}^{\mathrm{fix}, \mathrm{WE}}(p)$ (and no other duties) on weekends and public holidays each month.

\section{Soft constraints:}

Team staffing requirements

(25) At most abs $(g, t)$ physicians should be absent in each team $g \in G$ on any work day $t \in T \backslash\left(T^{\mathrm{WE}} \cup T^{\text {leave,work }}\right)$ that is not contained in a leave period.

(26) At most $\operatorname{abs}^{f}(g, t)$ fellows should be absent in each team $g \in G$ on any work day $t \in T \backslash\left(T^{\mathrm{WE}} \cup T^{\text {leave,work }}\right)$ that is not contained in a leave period.

(27) At most $\operatorname{abs}^{l}(g, t)$ physicians should be absent in each team $g \in G$ on any work day $t \in T^{\text {leave,work }}$ during a leave period.

(28) If the minimum required staffing of team $g \in G$ on a work day $t \in$ $T \backslash\left(T^{\mathrm{WE}} \cup T^{\text {leave,work }}\right)$ that is not contained in a leave period is at least two (i.e., if $\operatorname{size}(g)-\operatorname{abs}(g, t) \geq 2)$ and exactly size $(g)-\operatorname{abs}(g, t)$ physicians are present in team $g$ on day $t$ (counting possible float physicians assigned to $g$ on day $t$ ), then at most one physician in team $g$ should start late on day $t$. If less than $\operatorname{size}(g)-\operatorname{abs}(g, t)$ physicians are present, no physician should start late. 
(29) If the minimum required staffing of team $g \in G$ on a work day $t \in$ $T \backslash\left(T^{\mathrm{WE}} \cup T^{\text {leave,work }}\right)$ that is not contained in a leave period is less than two (i.e., if $\operatorname{size}(g)-\operatorname{abs}(g, t)<2)$ and at most $\operatorname{size}(g)-\operatorname{abs}(g, t)$ physicians are present in team $g$ on day $t$ (counting possible float physicians assigned to $g$ on day $t$ ), then no physician in team $g$ should start late on day $t$.

(30) At most one physician $p \in P_{g}$ should start late in each team $g \in G$ on any work day $t \in T^{\text {leave,work }}$ during a leave period (including possible float physicians assigned to $g$ on day $t$ ).

No (night) duty on the day after a night duty D4 or a late duty

(31) If physician $p \in P_{\mathrm{D} 4}$ is assigned the night duty D4 on day $t-1$, she should not be assigned any duty on day $t \in T$.

(32) If physician $p \in P_{\text {Late }}$ is assigned the late duty on day $t-1$, she should not be assigned a night duty on day $t \in T$.

Fair allocation of duties during leave periods

(33) Each physician $p \in P$ should be assigned at most one night duty in total during leave periods.

(34) If a physician $p \in P_{\text {Late }}$ in the duty group of the late duty is assigned at least one late duty during leave periods, she should not be assigned any night duties during leave periods.

Fair allocation of duties overall

(35) Each physician $p \in P \backslash\left(P^{\mathrm{fix}} \cup P^{\mathrm{fix}, \mathrm{WE}}\right)$ should be assigned at least $\left\lceil\sum_{d \in D_{p}}\left(\operatorname{nd}(p, d)+\operatorname{nd}^{\mathrm{WE}}(p, d)\right)\right\rceil-1$ duties in total.

(36) Each physician $p \in P \backslash\left(P^{\mathrm{fix}} \cup P^{\mathrm{fix}, \mathrm{WE}}\right)$ should be assigned at most $\left\lfloor\sum_{d \in D_{p}}\left(\operatorname{nd}(p, d)+\mathrm{nd}^{\mathrm{WE}}(p, d)\right)\right\rfloor+1$ duties in total.

Time between duties of the same physician

(37) The same physician $p \in P_{\text {Late }}$ that is assigned the late duty on day $t-1$ should also be assigned the late duty on day $t \in T$. 
(38) If a physician $p \in P$ is assigned a night duty $d \in D_{p} \backslash\{$ Late $\}$ on day $t \in T \backslash\{n-1, n\}$, she should not be assigned any night duty on day $t+2$.

(39) If a physician $p \in P$ is assigned a night duty $d \in D_{p} \backslash\{$ Late $\}$ on day $t \in T \backslash\{n-2, n-1, n\}$, she should not be assigned any night duty on day $t+3$.

We remark that, due to the number of physicians in the duty group of the late duty, each physician only has to perform at most two late duties on weekends during the whole planning period. Consequently, a physician cannot be assigned late duties on all days of two consecutive weekends without having a day off, even though this is not implemented as a constraint in the model.

\subsection{Handling of Unplanned Absences}

In addition to the duty roster, the model also generates an ordered list of possible substitutes for each duty on each day. This list is used to handle unplanned absences of physicians on duty (e.g., due to illness) that appear after the duty roster has been computed.

The list for duty $d \in D$ on day $t \in T$ consists of all physicians of the duty group of duty $d$ that could replace the physician who is currently assigned duty $d$ on day $t$. Here, a physician $p \in P_{d}$ is considered a feasible replacement for duty $d$ on day $t$ if $p$ is present on days $t$ and $t+1$, is not assigned any duty on day $t$ (and $t-1, t+1$ if $d \in\{\mathrm{D} 1, \mathrm{D} 2, \mathrm{D} 3, \mathrm{D} 5\}$ ), and assigning duty $d$ to $p$ on day $t$ does not yield any violations of team staffing or fellow staffing requirements. The list for duty $d$ and day $t$ is sorted so that physicians who either requested any duty on day $t$ (entry 1 ) or specifically requested duty $d$ on day $t$ appear at the beginning of the list and physicians that requested not to be assigned any duty on day $t$ (entry 3 ) appear at the end of the list. In case of an unplanned absence of the physician who is assigned duty $d$ on day $t$, the first physician on the list who is not also absent due to some unplanned event is then chosen as the replacement. Possible additional working hours resulting from this for the replacement physician are credited to her time account and compensated later on. 


\subsection{Implementation}

The input data for the model is read from an Excel file using a python script. The IP is then formulated within python using the PuLP modeling library [15] and solved using CBC 2.9 (an open-source MIP solver, see [16]) with the optimality gap set to $3 \%$. This value for the optimality gap was chosen since it leads to reasonable computation times while at the same time guaranteeing close to optimal solutions. Smaller values for the optimality gap (e.g., 1\%) turned out to significantly increase the computation times observed in practice without yielding any noticeable improvements in the quality of the resulting rosters.

After solving the IP, the resulting duty roster together with an overview of the assignment of float physicians to teams during work days is output as an Excel file. The lists of possible substitutes for each duty on each day are output as a separate Excel file. Additionally, a list containing all violations of team or fellow staffing requirements and all undesired duties in the resulting roster is output. All computations are performed on a standard laptop computer equipped with an Intel Core i7 mobile CPU (base frequency $2,3 \mathrm{GHz}$, max turbo frequency $3.5 \mathrm{GHz}$ ) and 16 gigabytes of main memory.

We note that the model is quite flexible with respect to changes of the input. For example, any length of the planning period is possible and the planning period does not have to start at the beginning of a month or with a specific day of the week. All data on the physicians, teams, and duty groups (including the numbers of physicians and teams) can also be changed easily in the input file.

\section{Results}

In this section, we analyze the performance of our rostering approach with respect to the quality of the resulting duty rosters and the computation time needed for generating the rosters. Moreover, we comment on the reception of the model and the generated duty rosters by the practice partner.

\subsection{Comparison to a Manually Generated Duty Roster}

For the first planning period after the implementation of the IP model, we worked in parallel to the scheduler of the hospital in order to compare the quality of the manually generated duty roster of the scheduler and the duty roster generated by the model (after the first planning period, only the ros-

ters produced by the model were used in practice and no manual rosters were 
generated anymore). For this planning period of seven weeks, duty rosters for 48 physicians (32 residents and 16 fellows) were generated. Three of the residents were float physicians, for which an assignment to teams was generated on all days where this was necessary in order to avoid violating team staffing requirements in some of the 12 teams. Due to special requirements, two of the physicians (one resident and one fellow) were excluded from the fair allocation rules (both during the week and for weekends / public holidays) and given a fixed number of duties of a given type per month.

Table 2 shows a comparison of the manually generated roster to the roster generated by the IP model (solved with the optimality gap set to 3\%) with respect to the most important metrics (violations of team / fellow staffing requirements, respected physician preferences, and fair distribution of duties). Moreover, the chosen objective function coefficients are given for the requirements that are represented as soft constraints in the model. These coefficients were chosen carefully in close cooperation with the department and the scheduler to reflect the department's priorities with respect to the generated rosters. An overview of all objective function coefficients used both for this comparison and by the practice partner is provided in Table 4 in the appendix.

As the results show, the duty roster generated by the IP model improves upon the manually generated roster in every single metric. While the manually generated duty roster leads to 11 and 5 additional violations of team and fellow staffing requirements, respectively, no additional violations of staffing requirements (in addition to the ones already existing due to absent physicians before the duties are assigned) occur in the roster generated by the model. Moreover, the model roster also respects the physicians' individual preferences better by fulfilling more duty requests and assigning no undesired duties. With respect to the fair allocation, the model roster respects all given bounds on the minimum and maximum numbers of duties to be assigned to each physician (both in each specific duty group and in total) since these are hard constraints of the model. In contrast, the manually generated roster does not achieve a fair distribution of duties among the physicians. Moreover, the manually generated roster assigns 20 duties in total to physicians from a wrong duty group (most likely in order to assign duties on days where only few physicians are available), which never happens in the duty roster generated by the model.

Overall, the duty roster generated by the IP model leads to an assignment 
of duties that greatly improves the team staffing levels while at the same time achieving a fair distribution of duties among the physicians and respecting the physicians' individual preferences better.

\subsection{Long-Term Results}

In order to evaluate the practical performance of our model over a longer time span, we analyze the duty rosters generated by the model on real input data from the department for four planning periods of two months each. Moreover, we report the computation times needed for generating the rosters.

Within each two-month planning period, a duty roster for about 50 physicians (about two thirds of which were residents) was generated. One or two of the residents were float physicians, for which an assignment to teams was generated on all days where this was necessary in order to avoid violating team staffing requirements in some of the 12 teams. The objective function coefficients were chosen as in Table 4 in the appendix.

Table 3 presents the most important metrics with respect to violations of team / fellow staffing requirements and respected physician preferences for each roster as well as the computation times.

Regarding the team staffing requirements, it can be observed that the model generates almost no additional violations of these constraints in periods 1-3. In period 4, several additional violations of team staffing and fellow staffing requirements occur. This is most likely due to the fact that this planning period included the summer holidays, where many physicians were on vacation (which can also be seen from the unusually large number of team and fellow staffing requirements that were already violated before assigning any duties). Hence, the general staffing as well as the fellow staffing of most teams were already close to their allowed minimum before the duties were assigned.

With respect to respecting the physicians' individual preferences, the model manages to fulfill between one third and half of all submitted duty requests within each planning period. Considering that quite often several physicians in the same duty groups request a duty on the same day (or even specifically request for the same duty), these fractions are rather high. Moreover, the model only assigns undesired duties very rarely.

The computation times needed for generating the rosters (including the time needed for reading the input files and for the preprocessing) vary between two and six minutes. Hence, the current solution method using an 


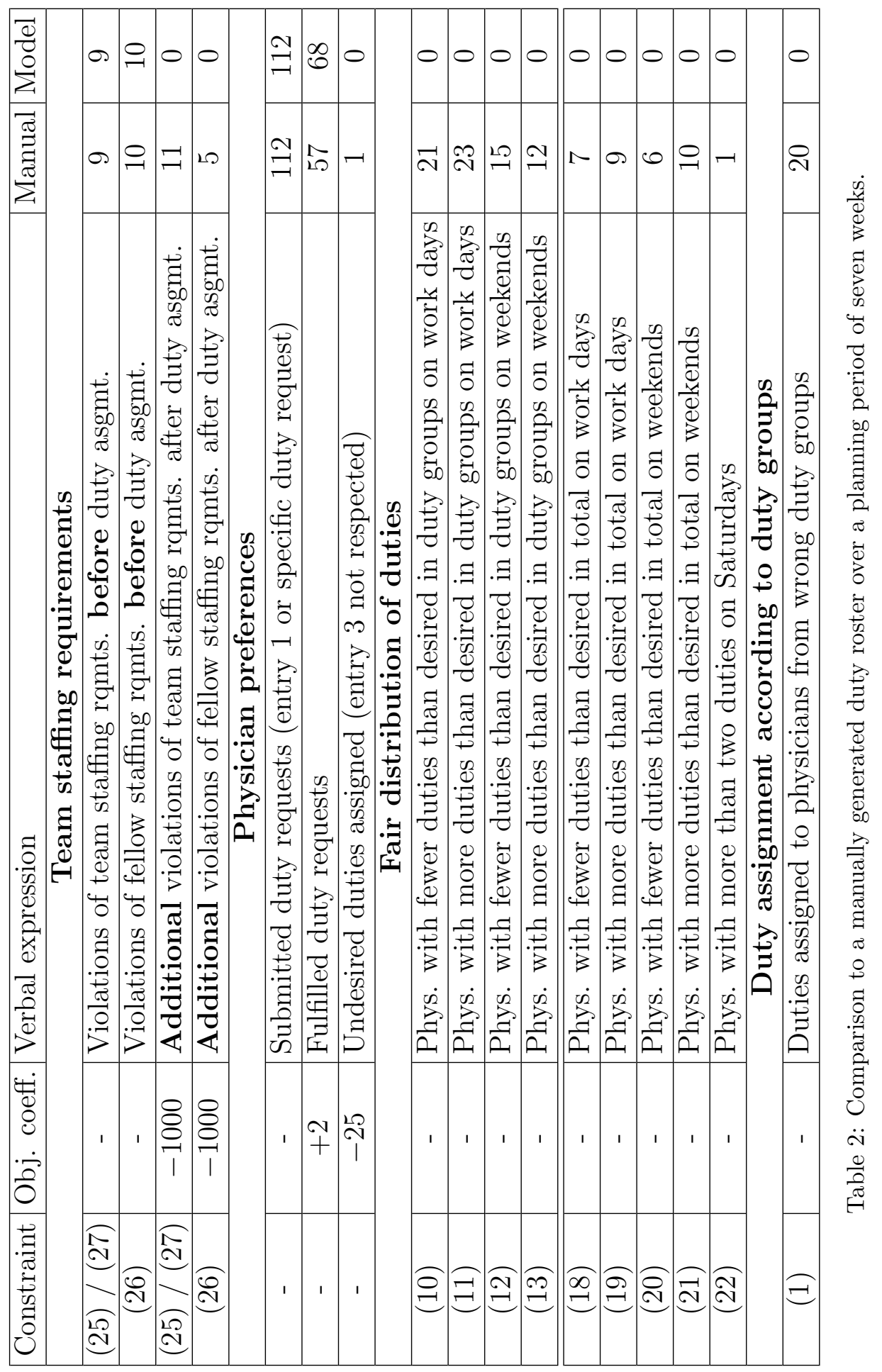


open-source MIP solver yields more than satisfactory computation times and there is no need for switching to a more powerful commercial solver.

\subsection{Reception by the Practice Partner}

The IP model and the generated rosters have been received very well by the practice partner. This is most likely due to the close collaboration with the scheduler and the department while designing the model. Early versions of the model were further developed in a constant feedback and adaption process in order to model the constraints and objectives of the department in the best possible way.

In particular, achieving a fair distribution of duties among the physicians has turned out to be extremely important for the department as well as for the individual physicians and lead to the integration of several additional constraints into the model (e.g., the upper bound of at most two duties on Saturdays for each physician). While a few physicians dislike our equal distribution approach (since they feel that they should be assigned less duties than their colleagues even though they are neither single parents nor on a part-time contract ${ }^{4}$ ), this approach to fairness is accepted very well by most of the physicians, who feel that the rosters generated by the model are fairer than the manually generated roster used before. Moreover, the department specifically prefers an equal distribution approach to other approaches we suggested (such as assigning a higher number of duties to physicians who request a large number of duties and a lower number of duties to physicians who request only few duties). The reason for this is that an equal distribution leads to an equal gain of experience by all physicians (e.g., due to handling of emergency patients arriving at night), which is considered important by the department especially since most of the scheduled physicians are residents.

In general, the reception of fairness in our study, where most physicians turned out to like (or even specifically demand) an equal distribution of duties, falls in line with the results of other case studies such as Bowers et al. [17], where a majority of the physicians preferred an equal distribution of workload to a nonequality roster that incorporates different preferences for specific duties / shifts.

\footnotetext{
${ }^{4}$ Single parents are usually excluded from the equal distribution as mentioned in Section 2 and part-time contracts are automatically taken into account in our approach as explained in Section 3.
} 


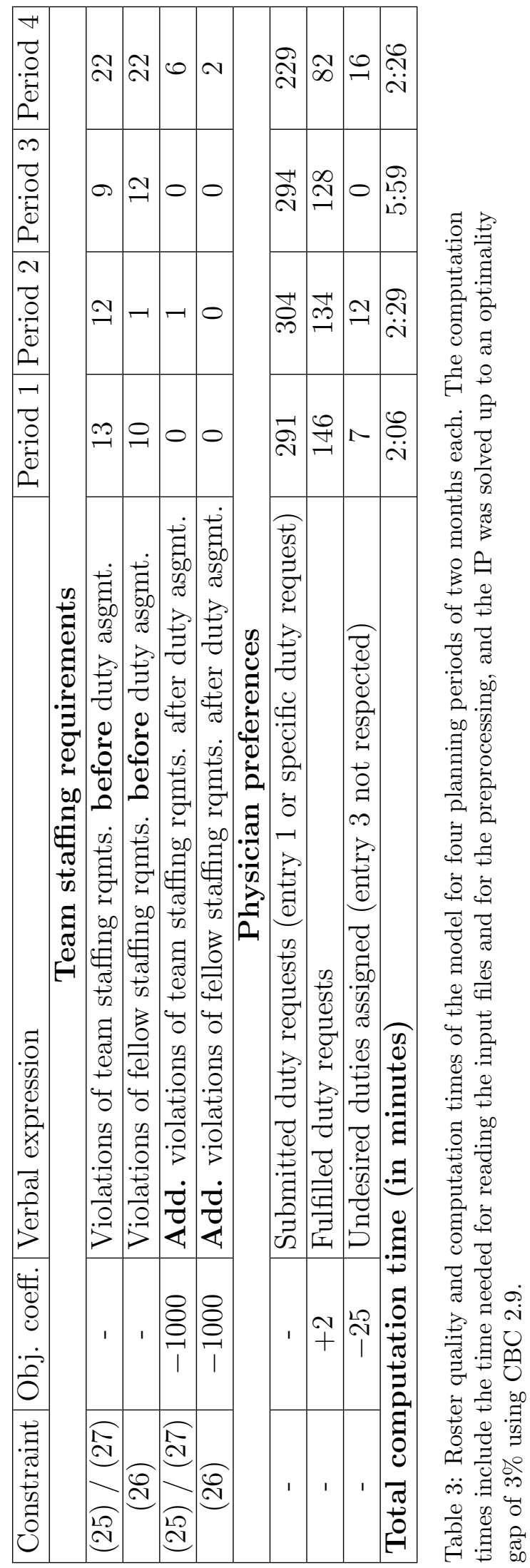


The lists of possible substitutes generated to handle unplanned absences of physicians on duty have turned out to be particularly useful in practice. In addition to finding replacements in cases where a physician on duty was absent on short notice (e.g., due to illness), these lists are also used frequently in case that minor adjustments to the duty roster have to be made after the final roster has been published to the physicians (e.g., because a physician has entered her absences incorrectly).

\section{Conclusion}

This paper presented a duty rostering problem faced at a department of orthopedics and trauma surgery and an IP model that has been used for solving the problem in practice for more than a year. A comparison of a duty roster generated by the IP model to a manually generated duty roster over a planning period of seven weeks showed that the IP model improves greatly upon the previously used manual rostering approach in all important metrics. Moreover, long-term results based on real world data revealed that the model is able to generate high-quality duty rosters fast even when using an open-source MIP solver.

Overall, we conclude that using an IP-based approach for generating duty rosters not only improved the quality of the rosters, but also helped to considerably reduce the time needed for generating the rosters. Moreover, due to the use of an open-source solver, the cost of implementing the model was quite low. Hence, our results demonstrate a large potential for using operations research methods in practical applications of physician scheduling.

Additionally, our approach included a first step towards a systematic handling of unforeseen events such as absences of physicians on duty by generating substitute lists for every duty on every day. These substitute lists have turned out to be very useful in practice and lead to better handling of unplanned absences of physicians without violating mandatory rest periods of the remaining physicians or staffing constraints. We believe that integrating even more sophisticated re-planning approaches into physician scheduling

models is a key point for increasing the usefulness of these models for practical applications. 


\section{Acknowledgment}

We are deeply grateful to the partner department and the scheduler for the great cooperation during the development of the duty rostering model presented in this paper and for letting us use their input data to test the model.

\section{References}

[1] M. W. Schall, T. Duffy, A. Krishnamurthy, O. Levesque, P. Metha, M. Murray, R. Parlier, R. Petzel, J. Sanderson, Improving patient access to the veterans health administrations primary care and specialty clinics, The Joint Commission Journal on Quality and Safety 30 (8) (2004) 415423.

[2] T. S. Bodenheimer, M. D. Smith, Primary care: Proposed solutions to the physician shortage without training more physicians, Health Affairs 32 (11) (2013) 1881-1886.

[3] M. Erhard, J. Schoenfelder, A. Fügener, J. O. Brunner, State of the art in physician scheduling, European Journal of Operational Research (online first), (2017) 1-18. doi:10.1016/j.ejor.2017.06.037.

[4] J. O. Brunner, G. Edenharter, Long term staff scheduling of physicians with different experience levels in hospitals using column generation, Health Care Management Science 14 (2) (2011) 189-202.

[5] J. Van den Bergh, J. Beliën, P. De Bruecker, E. Demeulemeester, L. De Boeck, Personnel scheduling: A literature review, European Journal of Operational Research 226 (3) (2013) 367-385.

[6] B. Cheang, H. Li, A. Lim, B. Rodrigues, Nurse rostering problems - a bibliographic survey, European Journal of Operational Research 151 (3) (2003) 447-460.

[7] E. K. Burke, P. De Causmaecker, G. Canden Berghe, H. Van Landeghem, The state of the art of nurse rostering, Journal of Scheduling 7 (6) (2004) 441-499.

[8] T. Benazzouz, A. Echchatbi, A. Bellabdaoui, A literature review on the nurses planning problems, International Journal of Mathematics and Computational Science 1 (5) (2015) 268-274. 
[9] M. W. Carter, S. D. Lapierre, Scheduling emergency room physicians, Health Care Management Science 4 (4) (2001) 347-360.

[10] A. Fügener, J. O. Brunner, A. Podtschaske, Duty and workstation rostering considering preferences and fairness: A case study at a department of anesthesiology, International Journal of Production Research 53 (24) (2015) 7465-7487.

[11] R. Stolletz, J. O. Brunner, Fair optimization of fortnightly physician schedules with flexible shifts, European Journal of Operational Research 219 (3) (2012) 622-629.

[12] C. N. Gross, A. Fügener, J. O. Brunner, Online rescheduling of physicians in hospitals, Flexible Services and Manufacturing Journal (2016) $1-33$.

[13] K. Riga, Mathematische Modelle für die Dienstplanung in Krankenhäusern, Bachelor's thesis, University of Kaiserslautern (January 2016).

[14] S. Helfrich, M. Volz, Modelle für die Dienstplanung in Krankenhäusern, Student project report, University of Kaiserslautern (September 2016).

[15] J. S. Roy, S. Mitchell, PuLP - A python Linear Programming API, https://github.com/coin-or/pulp.

[16] COIN-OR Initiative, CBC, https://projects. coin-or.org/Cbc.

[17] M. R. Bowers, C. E. Noon, W. Wu, J. K. Bass, Neonatal physician scheduling at the university of tennessee medical center, Interfaces 46 (2) (2016) 168-182. 


\section{Appendix}

We present the IP formulation of the model presented in Section 3.

Table 4 lists all decision variables and the corresponding objective function coefficients. All decision variables are either nonnegative integer variables or binary variables (for the binary variables, the meaning of the values 1 and 0 is described explicitly in the table). The value in brackets after each objective function coefficient corresponds to the standard value of this coefficient that is normally used by the practice partner. The (linear) objective function to be maximized is then given as the sum over all decision variables multiplied by their objective function coefficients (variables that do not appear in the objective function are marked by an entry "-" in the objective coefficient column in Table 4 ). The coefficient of each binary variable $x_{p, d, t}$ (which is set to 1 if and only if physician $p \in P$ is assigned duty $d \in D$ on day $t \in T$ ) depends on physician $p$ 's preference for duty $d$ on day $t$. It is set to $c^{\mathrm{dr}}$ (normally chosen as +2 ) if $p$ has requested duty $d$ on day $t$ (by using entry 1 or by specifically requesting duty $d$ ), to $c^{\text {undes }}$ (normally chosen as -100) if $p$ has marked day $t$ as undesired for being on duty (by using entry 3), and to zero, otherwise.

Since some of the constraints involve the assignment of duties on the last day of the previous planning period (day 0), this (fixed) assignment is provided in the input file and stored in the values $x_{d, p, 0}$ (which are, thus, not decision variables of the model).

Below, we present all constraints of the model partitioned into hard constraints, soft constraint, and auxiliary constraints. The auxiliary constraints

are used to set the values of certain variables and have no counterpart in the verbal description of the constraints provided in Section 3. 


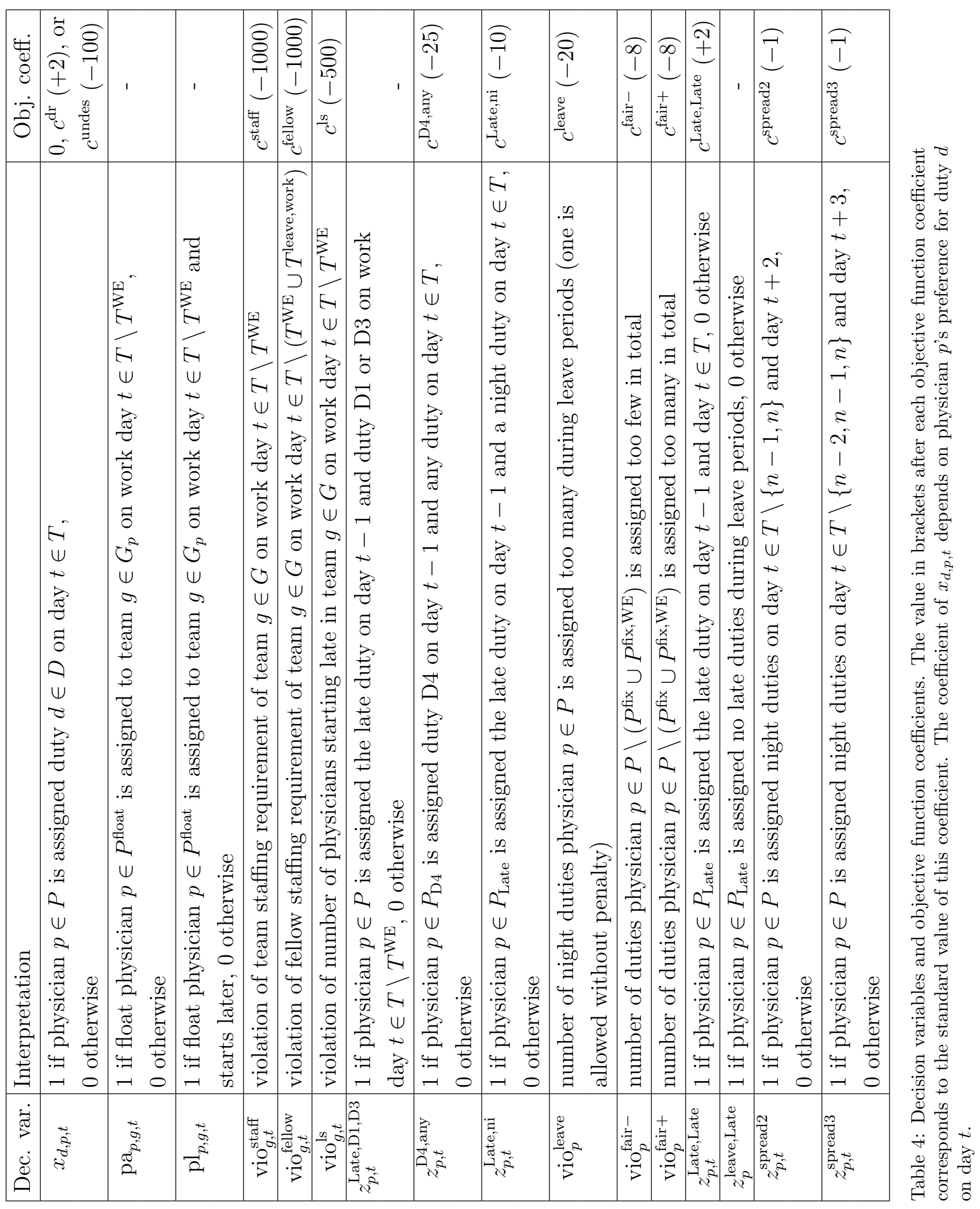




\section{Hard constraints:}

Assignment constraints

$$
\begin{aligned}
& \sum_{p \in P_{d}} x_{d, p, t}=1 \quad \forall d \in D, t \in T \\
& \sum_{d \in D_{p}} x_{d, p, t} \leq 1 \quad \forall p \in P, t \in T
\end{aligned}
$$

Respecting planned absences

$$
\begin{aligned}
(1-a(p, t)) & \geq \sum_{d \in D_{p}} x_{d, p, t} \quad \forall p \in P, t \in T \\
(1-a(p, t+1)) & \geq \sum_{d \in D_{p}} x_{d, p, t} \quad \forall p \in P, t \in T
\end{aligned}
$$

No duty on the day after a night duty (except for D4)

$$
\sum_{d \in D_{p} \backslash\{\text { D } 4, \text { Late }\}} x_{d, p, t}+\sum_{d \in D_{p}} x_{d, p, t+1} \leq 1 \quad \forall p \in P, t \in T \backslash\{n\}
$$

Assignment of float physicians to teams on work days

$$
\begin{array}{cl}
\sum_{g \in G_{p}} \mathrm{pa}_{p, g, t} \leq 1 \quad & \forall p \in P^{\mathrm{float}}, t \in T \backslash T^{\mathrm{WE}} \\
(1-a(p, t)) \geq \sum_{g \in G_{p}} \mathrm{pa}_{p, g, t} & \forall p \in P^{\mathrm{float}}, t \in T \backslash T^{\mathrm{WE}} \\
\sum_{g \in G_{p}} \mathrm{pa}_{p, t+1, g}+\sum_{d \in D_{p} \backslash\{\mathrm{D} 4, \mathrm{Late}\}} x_{d, p, t} \leq 1 & \forall p \in P^{\mathrm{float}}, t \in T \backslash\left(T^{\mathrm{WE}} \cup\{n\}\right) \\
\mathrm{pa}_{p, g, t} \geq i(p, g, t) & \forall p \in P^{\mathrm{float}}, t \in T \backslash T^{\mathrm{WE}}, g \in G_{p}
\end{array}
$$


Fair allocation of duties within each duty group

$$
\begin{aligned}
\sum_{t \in T \backslash T^{\mathrm{WE}+}} x_{d, p, t} \geq\lceil\mathrm{nd}(p, d)\rceil-1 & \forall d \in D \backslash\{\text { Late }\}, p \in P_{d} \backslash P^{\mathrm{fix}} \\
\sum_{t \in T \backslash T^{\mathrm{WE}+}} x_{d, p, t} \leq\lfloor\mathrm{nd}(p, d)\rfloor+1 & \forall d \in D \backslash\{\text { Late }\}, p \in P_{d} \backslash P^{\mathrm{fix}} \\
\sum_{t \in T^{\mathrm{WE}+}} x_{d, p, t} \geq\left\lceil\mathrm{nd}^{\mathrm{WE}}(p, d)\right\rceil-1 & \forall d \in D \backslash\{\text { Late }\}, p \in P_{d} \backslash P^{\mathrm{fix}, \mathrm{WE}} \\
\sum_{t \in T^{\mathrm{WE}+}} x_{d, p, t} \leq\left\lfloor\mathrm{nd}^{\mathrm{WE}}(p, d)\right\rfloor+1 & \forall d \in D \backslash\{\text { Late }\}, p \in P_{d} \backslash P^{\mathrm{fix}, \mathrm{WE}} \\
\sum_{t \in T \backslash T^{\mathrm{WE}}} x_{\text {Late }, p, t} \geq\lfloor\mathrm{nd}(p, \text { Late })\rfloor-1 & \forall p \in P_{\text {Late }} \backslash P^{\mathrm{fix}} \\
\sum_{t \in T \backslash T^{\mathrm{WE}}} x_{\text {Late }, p, t} \leq\lfloor\mathrm{nd}(p, \text { Late })\rfloor+2 & \forall p \in P_{\text {Late }} \backslash P^{\mathrm{fix}} \\
\sum_{t \in T^{\mathrm{WE}}} x_{\text {Late }, p, t} \geq\left\lfloor\mathrm{nd}^{\mathrm{WE}}(p, \text { Late })\right\rfloor-1 & \forall p \in P_{\text {Late }} \backslash P^{\mathrm{fix}, \mathrm{WE}} \\
\sum_{t \in T^{\mathrm{WE}}} x_{\text {Late }, p, t} \leq\left\lfloor\mathrm{nd}^{\mathrm{WE}}(p, \text { Late })\right\rfloor+2 & \forall p \in P_{\text {Late }} \backslash P^{\mathrm{fix}, \mathrm{WE}}
\end{aligned}
$$

Fair allocation of duties overall

$$
\begin{aligned}
& \sum_{d \in D_{p} \backslash\{\text { Late }\}} \sum_{t \in T \backslash T^{\mathrm{WE}}+} x_{d, p, t}+\sum_{t \in T \backslash T^{\mathrm{WE}}} x_{\text {Late }, p, t} \geq\left\lceil\sum_{d \in D_{p}} \operatorname{nd}(p, d)\right\rceil-2 \quad \forall p \in P \backslash P^{\mathrm{fix}} \\
& \sum_{d \in D_{p} \backslash\{\text { Late }\}} \sum_{t \in T \backslash T^{\mathrm{WE}}+} x_{d, p, t}+\sum_{t \in T \backslash T^{\mathrm{WE}}} x_{\text {Late }, p, t} \leq\left\lfloor\sum_{d \in D_{p}} \operatorname{nd}(p, d)\right\rfloor+2 \quad \forall p \in P \backslash P^{\mathrm{fix}} \\
& \sum_{d \in D_{p} \backslash\{\text { Late }\}} \sum_{t \in T^{\mathrm{WE}}+} x_{d, p, t}+\sum_{t \in T_{\mathrm{WE}}} x_{\text {Late }, p, t} \geq\left\lceil\sum_{d \in D_{p}} \mathrm{nd}^{\mathrm{WE}}(p, d)\right\rceil-1 \quad \forall p \in P \backslash P^{\mathrm{fix}, \mathrm{WE}} \\
& \sum_{d \in D_{p} \backslash\{\text { Late }\}} \sum_{t \in T^{\mathrm{WE}}+} x_{d, p, t}+\sum_{t \in T^{\mathrm{WE}}} x_{\text {Late }, p, t} \leq\left\lfloor\sum_{d \in D_{p}} \operatorname{nd}(p, d)^{\mathrm{WE}}\right\rfloor+1 \quad \forall p \in P \backslash P^{\mathrm{fix}, \mathrm{WE}}
\end{aligned}
$$


At most two duties on Saturdays for each physician

$$
\sum_{t \in T^{\mathrm{Sat}}} x_{d, p, t} \leq 2 \quad \forall p \in P \backslash P^{\mathrm{fix}, \mathrm{WE}}
$$

Numbers of duties of physicians with fixed numbers of duties

$$
\begin{aligned}
& \sum_{t \in T_{j} \backslash T^{\mathrm{WE}}+} x_{\operatorname{duty}^{\mathrm{fix}}(p), p, t}=\operatorname{nd}^{\mathrm{fix}}(p) \quad \forall p \in P^{\mathrm{fix}}: \operatorname{duty}^{\mathrm{fix}}(p) \neq \text { Late, } j \in\{1,2\} \\
& \sum_{t \in T_{j} \backslash T^{\mathrm{WE}}+} \sum_{d \in D_{p} \backslash\left\{\operatorname{duty}^{\mathrm{fix}}(p)\right\}} x_{d, p, t}=0 \quad \forall p \in P^{\mathrm{fix}}: \operatorname{duty}^{\mathrm{fix}}(p) \neq \text { Late, } j \in\{1,2\} \\
& \sum_{t \in T_{j} \backslash T^{\mathrm{WE}}} x_{\text {Late }, p, t}=\operatorname{nd}^{\mathrm{fix}}(p) \quad \forall p \in P^{\mathrm{fix}}: \operatorname{duty}^{\mathrm{fix}}(p)=\text { Late, } j \in\{1,2\} \\
& \sum_{t \in T_{j} \backslash T^{\mathrm{WE}}} \sum_{d \in D_{p} \backslash\{\text { Late }\}} x_{d, p, t}=0 \quad \forall p \in P^{\mathrm{fix}}: \operatorname{duty}^{\mathrm{fix}}(p)=\text { Late, } j \in\{1,2\} \\
& \sum_{t \in T_{j} \cap T^{\mathrm{WE}+}} x_{\mathrm{duty}^{\mathrm{fix}, \mathrm{WE}}(p), p, t}=\mathrm{nd}^{\mathrm{fix}, \mathrm{WE}}(p) \quad \forall p \in P^{\mathrm{fix}, \mathrm{WE}}: \text { duty }^{\mathrm{fix}, \mathrm{WE}}(p) \neq \text { Late, } j \in\{1,2\} \\
& \sum_{t \in T_{j} \cap T^{\mathrm{WE}+}} \sum_{d \in D_{p} \backslash\left\{\operatorname{duty}_{\mathrm{fix}, \mathrm{WE}}(p), \text { Late }\right\}} x_{d, p, t}=0 \quad \forall p \in P^{\mathrm{fix}, \mathrm{WE}}: \operatorname{duty}^{\mathrm{fix}, \mathrm{WE}}(p) \neq \text { Late, } j \in\{1,2\} \\
& \sum_{t \in T_{j} \cap T^{\mathrm{WE}}} x_{\text {Late }, p, t}=0 \quad \forall p \in P^{\mathrm{fix}, \mathrm{WE}}: \operatorname{duty}^{\mathrm{fix}, \mathrm{WE}}(p) \neq \text { Late, } j \in\{1,2\} \\
& \sum_{t \in T_{j} \cap T^{\mathrm{WE}}} x_{\text {Late }, p, t}=\mathrm{nd}^{\mathrm{fix}, \mathrm{WE}}(p) \quad \forall p \in P^{\mathrm{fix}, \mathrm{WE}}: \mathrm{duty}^{\mathrm{fix}, \mathrm{WE}}(p)=\text { Late, } j \in\{1,2\} \\
& \sum_{t \in T_{j} \cap T^{\mathrm{WE}}+} \sum_{d \in D_{p} \backslash\{\text { Late }\}} x_{d, p, t}=0 \quad \forall p \in P^{\mathrm{fix}, \mathrm{WE}}: \text { duty }^{\mathrm{fix}, \mathrm{WE}}(p)=\text { Late, } j \in\{1,2\}
\end{aligned}
$$




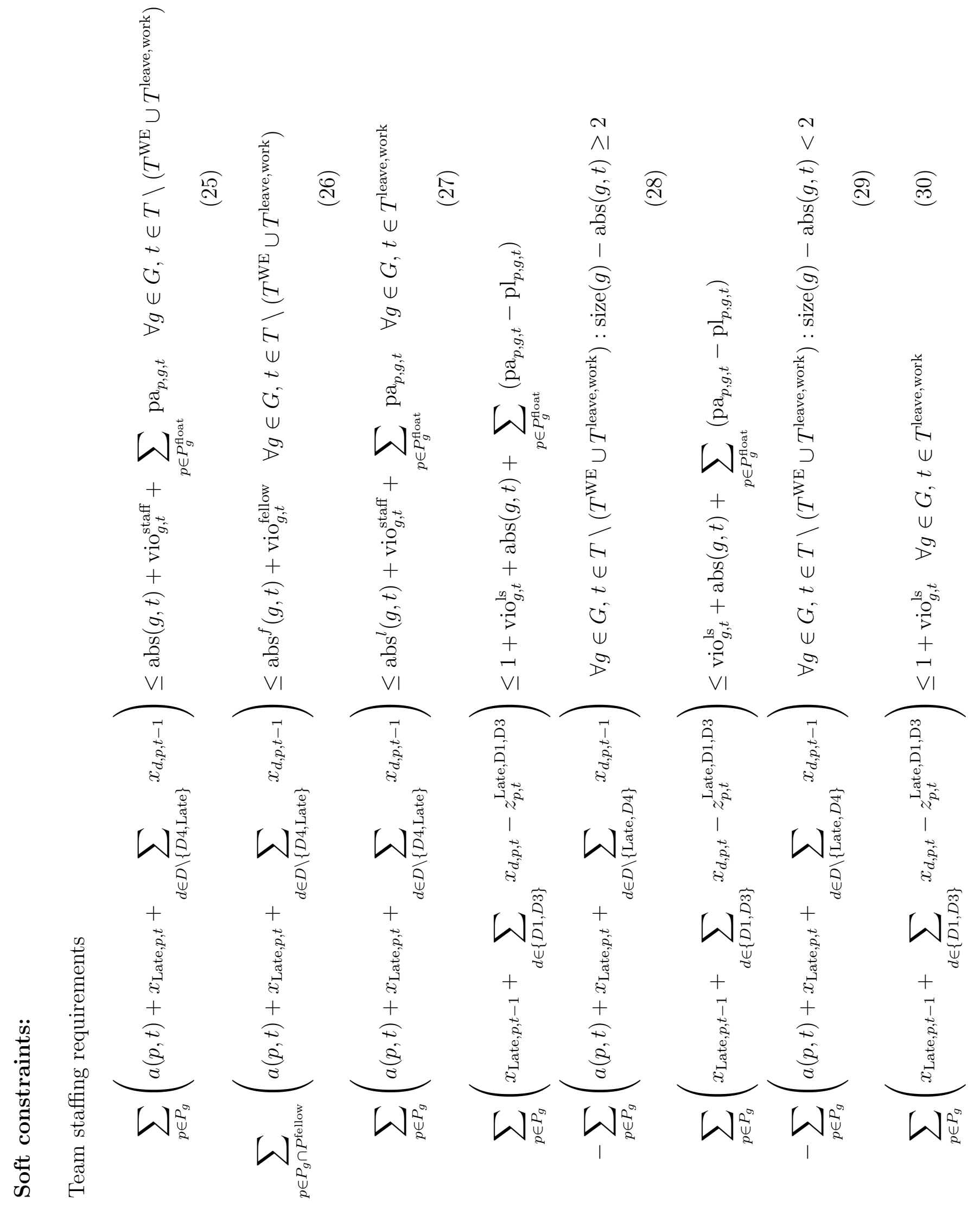


No (night) duty on the day after a night duty D4 or a late duty

$$
\begin{aligned}
x_{\mathrm{D} 4, p, t-1}+\sum_{d \in D_{p}} x_{d, p, t} \leq 1+z_{p, t}^{\mathrm{D} 4, \text { any }} & \forall p \in P_{\mathrm{D} 4}, t \in T \\
x_{\text {Late }, p, t-1}+\sum_{d \in D_{p} \backslash\{\text { Late }\}} x_{d, p, t} \leq 1+z_{p, t}^{\text {Late,ni }} & \forall p \in P_{\text {Late }}, t \in T
\end{aligned}
$$

Fair allocation of duties during leave periods

$$
\begin{array}{ll}
\sum_{t \in T^{\text {leave }}} \sum_{d \in D_{p} \backslash\{\text { Late }\}} x_{d, p, t} \leq 1+\text { vio }_{p}^{\text {leave }} & \forall p \in P \\
\sum_{t \in T^{\text {leave }}} \sum_{d \in D_{p} \backslash\{\text { Late }\}} x_{d, p, t} \leq z_{p}^{\text {leave,Late }}+\text { vio }_{p}^{\text {leave }} & \forall p \in P
\end{array}
$$

Fair allocation of duties overall

$$
\sum_{t \in T} \sum_{d \in D_{p}} x_{d, p, t} \geq\left\lceil\sum_{d \in D_{p}}\left(\operatorname{nd}(p, d)+\mathrm{nd}^{\mathrm{WE}}(p, d)\right)\right]-1-\operatorname{vio}_{p}^{\text {fair }-} \quad \forall p \in P \backslash\left(P^{\mathrm{fix}} \cup P^{\mathrm{fix}, \mathrm{WE}}\right)
$$

$$
\sum_{t \in T} \sum_{d \in D_{p}} x_{d, p, t} \leq\left\lfloor\sum_{d \in D_{p}}\left(\operatorname{nd}(p, d)+\operatorname{nd}^{\mathrm{WE}}(p, d)\right)\right\rfloor+1+\operatorname{vio}_{p}^{\text {fair }+} \forall p \in P \backslash\left(P^{\mathrm{fix}} \cup P^{\mathrm{fix}, \mathrm{WE}}\right)
$$

Time between duties of the same physician

$$
\begin{array}{rlrl}
z_{p, t}^{\text {Late,Late }} & \leq x_{\text {Late }, p, t-1} & & \forall p \in P_{\text {Late }}, t \in T \\
z_{p, t}^{\text {Late,Late }} & \leq x_{\text {Late }, p, t} & \forall p \in P_{\text {Late }}, t \in T \\
\sum_{d \in D \backslash\{\text { Late }\}}\left(x_{d, p, t}+x_{d, p, t+2}\right) & \leq 1+z_{p, t}^{\text {spread } 2} & & \forall p \in P, t \in T \backslash\{n-1, n\} \\
\sum_{d \in D \backslash\{\text { Late }\}}\left(x_{d, p, t}+x_{d, p, t+3}\right) & \leq 1+z_{p, t}^{\text {spread} 3} & & \forall p \in P, t \in T \backslash\{n-2, n-1, n\}
\end{array}
$$




\section{Auxiliary constraints:}

Determine values of variables $z_{p, t}^{\text {Late,D1,D3 }}$

$$
\begin{aligned}
x_{\text {Late }, p, t-1}+x_{\mathrm{D} 1, p, t}+x_{\mathrm{D} 3, p, t} & \leq 1+z_{p, t}^{\mathrm{Late}, \mathrm{D} 1, \mathrm{D} 3} & & \forall p \in P_{\text {Late }}, t \in T \backslash T^{\mathrm{WE}} \\
z_{p, t}^{\text {Late,D1,D3 }} & \leq x_{\text {Late }, p, t-1} & & \forall p \in P_{\text {Late }}, t \in T \backslash T^{\mathrm{WE}} \\
z_{p, t}^{\text {Late,D1,D3 }} & \leq x_{\mathrm{D} 1, p, t}+x_{\mathrm{D} 3, p, t} & & \forall p \in P_{\text {Late }}, t \in T \backslash T^{\mathrm{WE}}
\end{aligned}
$$

Determine values of variables $\mathrm{pl}_{p, g, t}$

$$
\begin{aligned}
& \mathrm{pl}_{p, g, t} \leq \mathrm{pa}_{p, g, t} \\
& \forall p \in P^{\text {float }}, g \in G_{p}, t \in T \backslash T^{\mathrm{WE}} \\
& \mathrm{pl}_{p, g, t} \leq x_{\text {Late }, p, t-1}+x_{\mathrm{D} 1, p, t}+x_{\mathrm{D} 3, p, t} \\
& \forall p \in P^{\text {float }}, g \in G_{p}, t \in T \backslash T^{\mathrm{WE}} \\
& 1+\mathrm{pl}_{p, g, t} \geq \mathrm{pa}_{p, g, t}+x_{\text {Late }, p, t-1} \\
& \forall p \in P^{\text {float }}, g \in G_{p}, t \in T \backslash T^{\mathrm{WE}} \\
& 1+\mathrm{pl}_{p, g, t} \geq \mathrm{pa}_{p, g, t}+x_{\mathrm{D} 1, p, t}+x_{\mathrm{D} 3, p, t} \\
& \forall p \in P^{\text {float }}, g \in G_{p}, t \in T \backslash T^{\mathrm{WE}}
\end{aligned}
$$

Determine values of variables $z_{p}^{\text {leave,Late }}$

$$
\begin{array}{rlrl}
\sum_{t \in T^{\text {leave }}} x_{\text {Late }, p, t} & \leq 200 \cdot\left(1-z_{p}^{\text {leave,Late }}\right) & \forall p \in P_{\text {Late }} \\
1-z_{p}^{\text {leave,Late }} \leq \sum_{t \in T^{\text {leave }}} x_{\text {Late }, p, t} & \forall p \in P_{\text {Late }}
\end{array}
$$

